\title{
Diagnosis of transthyretin cardiac amyloidosis with cadmium zinc telluride cameras: Is it feasible?
}

\author{
Roxana Campisi, $M D,{ }^{a, b}$ and Sonia S. Traverso, $M D^{b, c}$ \\ a Departments of Nuclear Medicine and Cardiovascular Imaging, Diagnóstico Maipú, Vicente \\ López, Buenos Aires, Argentina \\ b Department of Nuclear Medicine, Instituto Argentino de Diagnóstico y Tratamiento, Buenos \\ Aires, Argentina \\ c Departments of SPECT/CT and PET/CT, Fundación Centro Diagnóstico Nuclear, Buenos Aires, \\ Argentina
}

Received Jul 3, 2019; accepted Jul 5, 2019

doi: $10.1007 /$ s12350-020-02071-x

\section{See related article, pp. 371-380}

\section{CURRENT ROLE OF NUCLEAR CARDIOLOGY IN THE DIAGNOSIS OF CARDIAC AMYLOIDOSIS}

Cardiac amyloidosis is an infiltrative disease that results from the accumulation of misfolded protein deposits. The majority of cases of cardiac amyloidosis arise from two protein precursors: light-chain (AL) amyloidosis and transthyretin amyloidosis (ATTR). ${ }^{1}$ Amyloid in AL amyloidosis is derived from a misfolded monoclonal immunoglobulin light chain in the context of a plasma cell dyscrasia. Instead, ATTR amyloidosis results from accumulation of transthyretin protein, a serum transport protein for thyroid hormone and retinol that is produced by the liver. TTR amyloid is more frequently wild-type (ATTRwt) and acquired, but it may also be hereditary and associated with mutant forms of transthyretin. ${ }^{2}$ ATTRwt cardiac amyloid is also a disorder of aging individuals, ${ }^{1}$ with a prevalence of $17 \%$ in patients with heart failure with preserved ejection fraction on autopsy and a substantial male predominance. $^{3}$

Reprint requests: Roxana Campisi, MD, Departments of Nuclear Medicine and Cardiovascular Imaging, Diagnóstico Maipú, Av. Maipú 1668, B1602ABQ Vicente López, Buenos Aires, Argentina; roxanacampisi@yahoo.com

J Nucl Cardiol 2020;27:381-5.

$1071-3581 / \$ 34.00$

Copyright (C) 2020 American Society of Nuclear Cardiology.
The amyloid cardiomyopathy has high morbidity and mortality, but has emerged as an underdiagnosed and potentially treatable condition. ${ }^{3}$ The diagnosis is frequently delayed or missed due to a combination of factors comprised by a limited awareness and low suspicion levels, a suboptimal specificity of echocardiography, and the traditional requirement for histological confirmation in a tissue biopsy. ${ }^{4}$ Accurate typing of amyloid is essential since chemotherapy directed toward the AL amyloidosis can prolong life and new therapies for systemic ATTR have been approved by FDA. ${ }^{5}$ In patients with ATTR cardiomyopathy, a recent large randomized trial showed that treatment with tafamidis reduced all-cause mortality and cardiac hospitalization as well as improvement in exercise capacity and quality of life as compared with placebo, particularly amid early stages of the disease. ${ }^{6}$

Radionuclide imaging plays a critical role in the diagnosis and identification of cardiac ATTR amyloidosis. In fact, high sensitivity and specificity of Technetium (Tc) bone compounds scintigraphy for cardiac ATTR amyloidosis have been confirmed in several studies. ${ }^{7}$ Imaging techniques in nuclear medicine with specific radiotracers that bind to bone matrix components are the only modality that can accurately diagnose ATTR-type cardiomyopathy without the need for an invasive endomyocardial biopsy in patients without evidence for monoclonal proteins in serum and urine. ${ }^{4}$

There are three different bone molecules that have varying avidity for cardiac amyloid deposits that include Tc-99m-labeled agents pyrophosphate (Tc-99m PYP), hydroxymethylenediphosphonate (Tc-99m HMDP), and 3,3-diphosphono-1,2-propanodicarboxylic acid (Tc-99m 
DPD). Although the mechanism of binding to amyloid is not completely clear, it is believed that they are probably taken up via calcium-mediated mechanism. ${ }^{8}$ The first reports of bone scan with increased Tc-99m PYP uptake in the heart of patients with amyloid infiltration date from the early $1980 .{ }^{9,10}$ These radiotracers could be used to differentiate ATTR from AL amyloidosis with a high sensitivity and specificity as they have an affinity for the amyloid fibrils in ATTR amyloidosis, ${ }^{11}$ (Figure 1). Most experiences in imaging protocols for Tc99m PYP, DPD, and HMDP are with planar imaging followed by SPECT if planar is positive. Planar imaging alone is limited because myocardial uptake cannot be discerned from vascular blood pool uptake. The timing of imaging after injection of radiotracer varies from 1 to 3 hours. Most experience with Tc-99m PYP protocols is at 1 or 3 hour and for Tc99m DPD, HMDP at 3 hours. $^{7}$

Quantitation of Tc-99m compounds uptake (PYP, DPD, HMDP) is key in the diagnosis of cardiac ATTR amyloidosis and can be performed either visually or by quantitative analysis:

1. Semi-quantitative analysis: This approach involves the visual comparison of myocardial to bone (rib) uptake at 3 hours as described by Perugini and colleagues ${ }^{11}$ : Grade 0: no myocardial uptake and normal bone uptake; Grade 1: myocardial uptake less than bone uptake; Grade 2: myocardial uptake equal to bone uptake and Grade 3: myocardial uptake greater than bone uptake with mild/absent rib uptake. Visual scores of greater than or equal to 2 on planar or SPECT images are classified as ATTR positive and scores less than 2 as ATTR negative. ${ }^{12}$

2. Quantitative analysis: In order to distinguish ATTR from $\mathrm{AL}$ amyloidosis, Bokhari and colleagues ${ }^{13}$ described a myocardial-to-contralateral lung ratio of uptake at 1 hour on planar imaging. Circular target regions of interest (ROI) are drawn over the heart in the planar and are mirrored over the contralateral chest to account for background and ribs. Total and absolute mean counts are measured in each ROI. Then, a heart-to-contralateral (H/CL) ratio is calculated as the fraction of heart ROI mean counts to CL chest ROI mean counts. H/CL ratios of $\geq 1.5$ at 1 hour are classified as ATTR positive and ratios less than 1.5 as ATTR negative. For 3 hours of imaging, the cut-off value validated for ATTR positive is considered $\geq 1.3 .^{14}$

ASNC Practice Points document on the use of Tc99m PYP in diagnosing ATTR recommends the interpretation of the findings into categories are as follows ${ }^{12}$ :

1. Not suggestive: A semi-quantitative score of 0 or $\mathrm{H} /$ CL ratio less than 1.

2. Strongly suggestive: A semi-quantitative score of 2 or 3 or $\mathrm{H} / \mathrm{CL}$ ratio more than 1.5 .

3. Equivocal: A semi-quantitative score of 1 or $\mathrm{H} / \mathrm{CL}$ ratio $1-1.5$

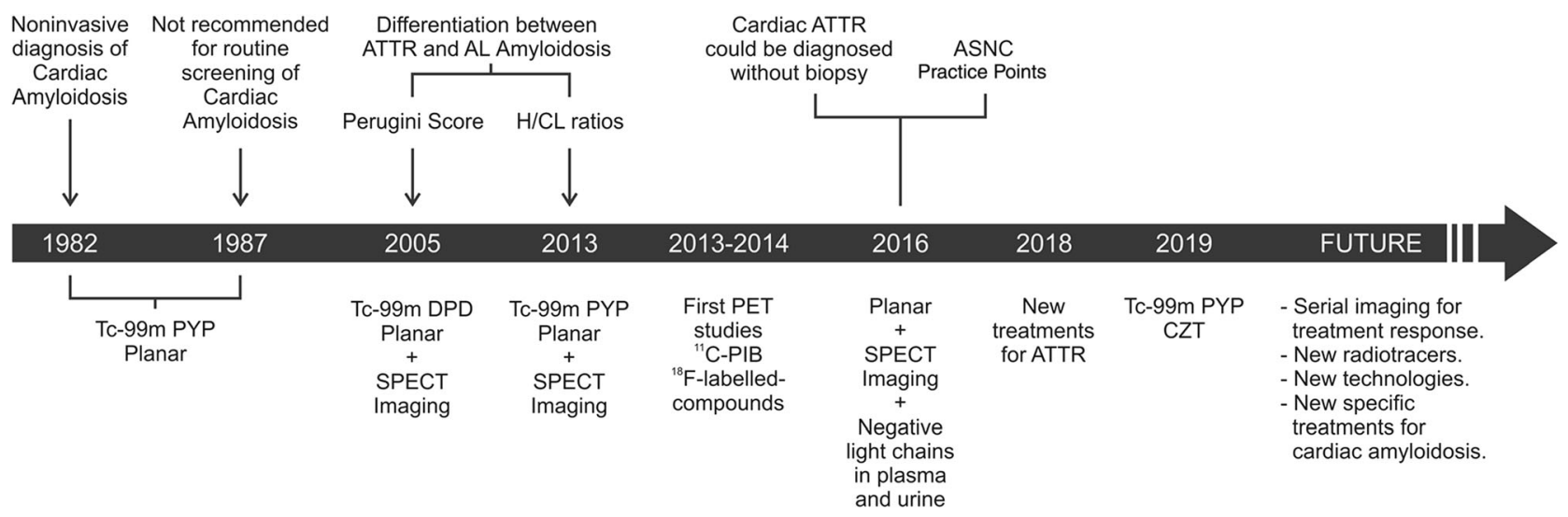

Figure 1. A timeline of nuclear imaging techniques in the diagnosis of cardiac amyloidosis. Sensitivity and specificity of Tc-99m bone avid compounds for differentiation between ATTR and $\mathrm{AL}$ types increased with the incorporation of semi-quantitative and quantitative analysis and SPECT imaging. Of clinical relevance, cardiac ATTR amyloidosis can now be diagnosed by Tc$99 \mathrm{~m}$ bone-avid radiotracers without the need of endomyocardial biopsy. Since new therapies are available for treating ATTR, early detection is critical. Future perspectives are depicted. ATTR, Transthyretin amyloidosis; $A L$, Immunoglobulin light-chain amyloidosis; $H / C L$, Heart-to-contralateral lung; ASNC, American Society of Nuclear Cardiology; Tc-99m PYP, 99m-technetiumlabeled pyrophosphate; Tc-99m DPD, 99m-technetium-labeled 3,3-diphosphono-1,2-propanodicarboxylic acid; SPECT, Single-Photon Emission Computed Tomography; PET, Positron Emission Tomography; ${ }^{11} C$-PIB,${ }^{11} \mathrm{C}$-Pittsburgh Compound-B; CZT, Cadmium Zinc Telluride. 
Of note, cardiac uptake of Tc-99m bone compounds can occur in a small proportion of patients with $\mathrm{AL}$ amyloidosis, usually in the category of equivocal study, thus leading to a challenging discrimination between early ATTR and AL cardiac amyloidosis. ${ }^{4}$ In such scenario, further evaluation including histological diagnosis is currently recommended. ${ }^{12}$ Amyloid-binding positron emission tomography (PET) tracers $\left({ }^{11} \mathrm{C}\right.$-Pittsburgh Compound-B (PIB), ${ }^{18} \mathrm{~F}$-florbetaben, and ${ }^{18} \mathrm{~F}$ florbetapir) are highly specific to image amyloid deposits and bind both AL and ATTR cardiac amyloidosis and therefore could be considered as an alternative in these patients. ${ }^{15,16}$ Despite preliminary experience with these tracers demonstrated high sensitivity for detection of cardiac amyloidosis, they did not allow clear differentiation between AL and ATTR types.

\section{DIAGNOSING CARDIAC AMYLOIDOSIS WITH CADMIUM ZINC TELLURIDE IMAGING}

In this edition of the Journal, Flaherty et $\mathrm{al}^{17}$ compared the sensitivity and specificity of planar and cadmium zinc telluride (CZT) imaging [Discovery NM530c] of Tc-99m PYP for the diagnosis of cardiac ATTR amyloidosis. The study population comprised 43 patients that were imaged the same day with Tc-99m PYP planar and a CZT camera. The clinical diagnostic criteria for ATTR $(n=24)$ was defined by (1) endomyocardial biopsy 29\%, (2) documented amyloidogenic TTR mutation by DNA analysis and echocardiographically defined evidence of amyloid cardiomyopathy (thickness of left ventricular septum or posterior wall of $>1.2 \mathrm{~cm}$ without another cause of left ventricular hypertrophy) without evidence of plasma cell dyscrasia $4 \%$, or (3) planar Tc-99m PYP indicative of ATTR $(\mathrm{H} /$ CL ratio $\geq 1.5$ and semi-quantitative score $\geq 2$ ) and echocardiographically defined evidence of amyloid cardiomyopathy without evidence of plasma cell dyscrasia $67 \%$. Of these ATTR-positive patients, 9\% were classified as ATTRwt. Of the 19 non-ATTR patients, 3 were classified as AL amyloidosis and 16 as heart failure with preserved ejection fraction.

Planar images were obtained at 1 hour post-Tc-99m PYP injection. Following planar imaging, patients immediately underwent CZT scintigraphy. Myocardial Tc-99m PYP uptake was quantified by H/CL ratios. The 8th, 9th, or 10th of the 19-projection holes on CZT scans were the most visually similar to an anterior planar view, and the most clear hole was analyzed quantitatively. Due to the decreased field of view (FOV) on CZT imaging relative to the target $\mathrm{ROI}$, the background was halved in size rather than mirrored (as performed in Anger cameras). A $\mathrm{H} / \mathrm{CL}$ ratio of $\geq 1.5$ indicated ATTR positive. A semi-quantitative visual analysis was also performed to assess Tc-99m PYP uptake on planar scans. Because bone uptake is not visible on CZT imaging, CZT scans were not assessed semi-quantitatively. Using the previously validated diagnostic threshold $(\mathrm{H} / \mathrm{CL} \geq 1.5)$, sensitivity and specificity of CZT scintigraphy was measured. Among subjects, there was no significant difference in the proportion of ATTRpositive identification between the two imaging modalities. There was a high linear correlation between CZT and planar H/CL ratios $(\mathrm{r}=.92, \mathrm{p}<.0001)$, and low inter- $($ ICC $=0.89(0.80-0.94))$ and intra- $($ ICC $=0.80$ (0.65-0.89)) observer variability for all groups. Sensitivity and specificity for the diagnosis of ATTR was $100 \%$ using CZT H/CL ratios with a 1.5 cut-off in patients with cardiac amyloidosis on biopsy or per noninvasive diagnostic criteria $(\mathrm{n}=29)$. The authors concluded that CZT cameras are an accurate and valid imaging modality to diagnose ATTR cardiac amyloidosis. However, the authors acknowledge that due to the limited FOV provided by CZT cameras, it decreased the image quality of views most similar to anterior planar views and as such accounts for the 7\% of scans that were unusable in the study. Further research is needed for solutions in image quality with these systems. It is also important to the remark that accurate localization of Tc$99 \mathrm{~m}$ PYP with these cameras is difficult when the myocardial uptake is low. ${ }^{18}$ In this scenario, cardiac computed tomography (CT) images can be useful. SPECT/CT would improve the definition of the myocardial boundaries and would provide attenuation-corrected images for absolute quantitation of Tc-99m PYP uptake in these patients. Alternatively, as recently suggested by Tamarappoo et al., simultaneous Tc-99m/Tl-201 dualisotope SPECT imaging in patients with suspected cardiac amyloidosis would afford accurate localization of the heart. ${ }^{18}$

Nuclear cardiac imaging has progressed from planar scintigraphy to SPECT imaging, leading to the development of CZT cameras. For cardiac SPECT imaging, studies have shown that CZT cameras yield high-quality images while allowing for decreased radioisotope doses and imaging time. ${ }^{19}$ Because of these characteristics, CZT cameras are increasingly used in clinical practice for SPECT myocardial perfusion imaging. For detection of cardiac ATTR amyloidosis, conventional SPECT imaging is routinely performed in most laboratories along with planar images or acquired when planar is positive. ${ }^{7,18}$ SPECT is increasingly used in this clinical setting because it eliminates contamination from overlying rib and therefore aids in distinguishing residual blood pool activity from myocardial uptake. ${ }^{12,18}$ SPECT imaging is the only manner to perform cardiac studies in CZT cameras. Flaherty and colleagues ${ }^{17}$ propose for the first time a quantitative methodology to diagnose 
patients with cardiac ATTR using CZT Tc-99m PYP scintigraphy. The quantitative methodology utilized in their study with a CZT camera is built on the previously established Tc-99m PYP planar method of using H/CL ratios. It is important to mention that this is a singlecenter study and findings of this study are only applicable to Tc-99m PYP-tracer/CZT [Discovery NM530c] imaging in patients with advanced symptoms of ATTR. Therefore, these study findings should not be extrapolated to patients at the early stages of ATTR or other CZT systems until further research is accomplished.

\section{FUTURE PERSPECTIVES}

Differentiation between the types of cardiac amyloidosis is important for prognosis, therapy, and genetic counseling. ${ }^{12}$ Since cardiac amyloidosis is now a potentially treatable disease, early diagnosis is a key factor for improving survival and quality of life. Compelling evidence shows that radionuclide imaging plays a critical role in the non-invasive diagnosis of cardiac ATTR. ${ }^{7,20}$ Due to the high sensitivity and specificity, Tc99m bone-avid radiotracers myocardial uptake are now considered sufficient to establish diagnosis of cardiac ATTR, obviating the need for endomyocardial biopsy. ${ }^{4}$ For patients with AL amyloidosis, Tc99m bone compound scintigraphy is less sensitive. Amyloid-binding tracers along with PET/CT imaging are promising, but still in the research field. ${ }^{7}$

Planar imaging is the most commonly employed method for diagnosis of cardiac ATTR. Conventional SPECT imaging is not required for identification of cardiac ATTR by the guidelines. ${ }^{12}$ However, SPECT imaging is increasingly being utilized in clinical practice, in particular, in patients when planar images are positive. ${ }^{18} \mathrm{CZT}$ imaging is now in the current practice of nuclear cardiology. ${ }^{19}$ As technology progresses, it is likely the prevalence of CZT cameras will grow. Therefore, validation of protocols for image acquisition and analysis with these systems are needed for identification of patients with cardiac ATTR. We agree with Flaherty and colleagues ${ }^{17}$ that by growing the number of centers capable of using highly specific and sensitive modalities of diagnosis of cardiac amyloidosis, appropriate treatments and preventions can be implemented sooner to those patients who would benefit. As technology advances and new radiotracers will be clinically validated, patients with different types of cardiac amyloidosis will be identified non-invasively by radionuclide techniques.

\section{Acknowledgments}

The authors are thankful to Daniel Cirigliano for the artwork.

\section{Disclosures}

The authors have no conflicts of interest to declare

\section{References}

1. Treibel TA, Fontana M, Gilbertson JA, Castelletti S, Sk White, Scully R, et al. Occult transthyretin cardiac amyloid in severe calcific aortic stenosis. Circ Cardiovasc Imaging 2016;9:e005066.

2. Siddiqi O, Ruberg F. Cardiac amyloidosis: An update on pathophysiology, diagnosis, and treatment. Trends Cardiovasc Med 2018;28:10-21.

3. Mohammed SF, Mirzoyev SA, Edwards WD, Dogan A, Grogan DR, Dunlay SM, et al. Left ventricular amyloid deposition in patients with heart preserved ejection fraction. JACC Heart Fail 2014;2:113-22.

4. Guillmore JD, Maurer MS, Falk RH, Merlini G, Damy T, Dispenzieri A, et al. Nonbiopsy diagnosis of cardiac transthyretin amyloidosis. Circulation 2016;133:2404-12.

5. Solomon SD, Adams D, Kristen A, Grogan M, González-Duarte A, Maurer MS, et al. Effects of patisiran, an RNA interference therapeutic, on cardiac parameters in patients with hereditary transthyretin-mediated amyloidosis. Circulation 2019;139:431-43.

6. Maurer MS, Schwartz JH, Gundapaneni B, Elliott PM, Merlini G, Waddington-Cruz $\mathrm{M}$, et al. Tafamidis treatment for patients with transthyretin amyloid cardiomyopathy. N Engl J Med 2018;379: 1007-16.

7. Singh V, Falk R, Di Carli MF, Kijewski M, Rapezzi C, Dorbala S. State-of-the-art radionuclide imaging in cardiac transthyretin amyloidosis. J Nucl Cardiol 2019;26:158-73.

8. Falk RH, Quarta CC, Dorbala S. How to image cardiac amyloidosis. Circ Cardiovasc Imaging 2014;7:553-62.

9. Wizenberg TA, Muz J, Sohn YH, Samlowski W, Weissler AM. Value of positive myocardial technetium-99m-pyrophosphate scintigraphy in the noninvasive diagnosis of cardiac amyloidosis. Am Heart J 1982;103:468-73.

10. Gertz MA, Brown ML, Hauser MF, Kyle RA. Utility of technetium Tc $99 \mathrm{~m}$ pyrophosphate bone scanning in cardiac amyloidosis. Arch Intern Med 1987;147:1039-44.

11. Perugini E, Guidalotti PL, Salvi F, Cooke RM, Pettinato C, Riva $\mathrm{L}$, et al. Noninvasive etiologic diagnosis of cardiac amyloidosis using 99mTc-3,3-diphosphono-1,2-propanodicarboxylic acid scinitigraphy. J Am Coll Cardiol 2005;46:1076-84.

12. Dorbala S, Bokhari S, Miller E, Bullock-Palmer RP, Soman P, Thompson R. American Society of Nuclear Cardiology (ASNC) practice points on technetium-99m pyrophosphate imaging for transthyretin cardiac amyloidosis-1st edition in 2016, updated in 2019.

13. Bokhari S, Castaño A, Pozniakoff T, Deslisle S, Latif F, Maurer MS. (99m)Tc-pyrophosphate scintigraphy for differentiating lightchain cardiac amyloidosis from the transthyretin-related familial and senile cardiac amyloidoses. Circ Cardiovasc Imaging 2013:6:195-201. 
14. Castaño A, Haq M, Narotsky DL, Goldsmith J, Weinberg RL, Morgenstern R, et al. Multicenter study of planar technetium 99m pyrophosphate cardiac imaging. Predicting survival for patients with ATTR cardiac amyloidosis. JAMA Cardiol 2016;1:880-9.

15. Lee S-P, Lee ES, Choi H, Im HJ, Lee MH, et al. 11C-Pittsburgh B PET imaging in cardiac amyloidosis. JACC Cardiovasc Imaging 2015;8:50-9.

16. Dorbala S, Vangala D, Semer J, Strader C, Bruyere JR, Di Carli MF, et al. Imaging cardiac amyloidosis: A pilot study using 18Fflorbetapir positron emission tomography. Eur J Nucl Med Mol Imaging 2014;41:1652-62.

17. Flaherty K, Morgenstern R, Pozniakoff T, DeLuca A, Castaño A, Maurer M, et al. 99mTechnetium pyrophosphate scintigraphy with cadmium zinc telluride cameras is a highly sensitive and specific imaging modality to diagnose tarnsthyretin cardiac amyloidosis. J Nucl Cardiol 2019. https://doi.org/10.1007/s12350-019-01831-8.
18. Tamarapoo B, Otaki Y, Manabe O, Hyun M, Cantu S, Anson Y, et al. Simultaneous Tc-99m PYP/Tl-201 dual-isotope SPECT myocardial imaging in patients with suspected cardiac amyloidosis. J Nucl Cardiol 2019. https://doi.org/10.1007/s12350-01901753-5.

19. Acampa W, Buechel RR, Gimelli A. Low dose in nuclear cardiology: State of the art in the era of new cadmium-zinc-telluride cameras. Eur Heart J 2016;17:591-5.

20. Bullock-Palmer RP. Diagnosing cardiac amyloidosis: A wealth of new possibilities with nuclear cardiac imaging. J Nucl Cardiol 2019. https://doi.org/10.1007/s12650-019-01740-w.

Publisher's Note Springer Nature remains neutral with regard to jurisdictional claims in published maps and institutional affiliations. 